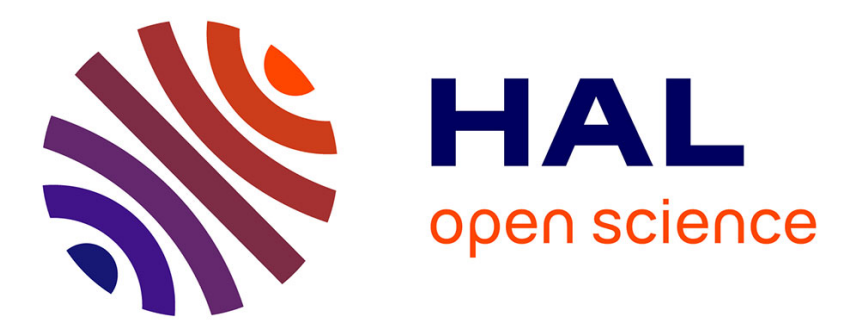

\title{
An Introduction to PHM and Prognostics Case Studies Hong-Bae Jun
}

\section{To cite this version:}

Hong-Bae Jun. An Introduction to PHM and Prognostics Case Studies. IFIP International Conference on Advances in Production Management Systems (APMS), Aug 2018, Seoul, South Korea. pp.304-310, 10.1007/978-3-319-99707-0_38. hal-02177891

\section{HAL Id: hal-02177891 \\ https://hal.inria.fr/hal-02177891}

Submitted on 9 Jul 2019

HAL is a multi-disciplinary open access archive for the deposit and dissemination of scientific research documents, whether they are published or not. The documents may come from teaching and research institutions in France or abroad, or from public or private research centers.
L'archive ouverte pluridisciplinaire HAL, est destinée au dépôt et à la diffusion de documents scientifiques de niveau recherche, publiés ou non, émanant des établissements d'enseignement et de recherche français ou étrangers, des laboratoires publics ou privés.

\section{(c)(1)}

Distributed under a Creative Commons Attribution| 4.0 International License 


\title{
An introduction to $\mathrm{PHM}$ and prognostics case studies
}

\author{
Hong-Bae Jun \\ Hongik University, Dept. of Industrial Engineering, Seoul 04066, South Korea \\ hongbae@gmail.com
}

\begin{abstract}
Large-scale systems such as plants should be operated with high reliability and availability because the downtime due to system failure has a significant influence on the manufacturing activity. Nowadays the emerging ICTs (Information Communication Technologies) and sensor technologies make it possible to monitor health status of important equipment of the plant and do diagnosis and prognosis for further suitable maintenance actions in real time, which leads to much concern on PHM (Prognostics and Health Management) or CBM (Condition-Based Maintenance). In this study, we briefly look through the concept of PHM and introduce prognostics case studies for plant equipment.
\end{abstract}

Keywords: Prognostics and Health Management, Condition-based maintenance, Plant equipment, Maintenance, Prognostics

\section{Introduction}

In general, maintenance is defined as all technical and managerial actions taken during usage period to maintain or restore the required functionality of a product or an asset. There have been various classifications of maintenance policies [1]. Simply, maintenance policies can be divided into breakdown maintenance and preventive maintenance. In the breakdown maintenance, the maintenance action is taken after some problems such as breakdowns in a product are found while the preventive maintenance periodically checks a product with a certain interval in order to prevent the abnormality of the product. The preventive maintenance is again classified into TBM (Time-based maintenance) and CBM (Condition-based maintenance) depending on the criteria for determining maintenance action; fixed time or mileage for TBM and product status for CBM. Recently, PHM (Prognostics and Health Management), the similar concept with CBM, has been highlighted with the concepts of Industry 4.0 and smart factory.

Large-scale systems such as plants should be operated with high reliability and availability because the downtime due to system failure has a significant influence on manufacturing activities. To meet such a demand, reliable and robust methods for monitoring, diagnosing, and predicting the abnormal status of important equipment, and making decision for appropriate maintenance actions are required. These are the core of PHM. 
Recently, advanced IoT (Internet Of Things) technologies including internetaware smart senors [2], embedded system [3], cloud-assisted IWN (Industrial Wireless Network) [3], WSNs (Wireless Sensor Networks) [4], RFID (Radio Frequency Identification), and SCADA (Supervisory Control And Data Acquisition) are expected to be rapidly used for gathering and monitoring the status data of plant equipment during their usage period. In this environment, capturing the status of plant equipment related to performance, operation and environmental conditions, and detecting their anomalies in a real-time way become challenging issues. The raw data from sensors need to be efficiently managed and transformed to usable information through data fusion, which in turn must be converted to predictive insights via knowledge discovery, ultimately facilitating automated or human-induced tactical decisions or strategic maintenance policy.

Though the data gathering systems are becoming relatively mature, a lot of innovative research need to be done on knowledge discovery from huge repositories of plant data. In this vein, recently the importance of PHM has been highlighted and tried to be implemented in various domains. In order to apply the concept of PHM into current maintenance policy and implement the PHM system, first and foremost, it is prerequisite to understand what PHM is. In this study, we briefly look through the concept of PHM and related previous works. Then, we introduce prognostics case studies for three plant equipment.

\section{PHM}

In recent years, PHM has emerged as one of the key enablers for achieving efficient system-level maintenance and lowering life-cycle costs need $[5,6]$. It could improve availability of critical engineering assets while reducing inopportune spending and security risks [7]. The IEEE Reliability Society (www.phmconf.org) defines PHM as "a system engineering discipline focusing on detection, prediction, and management of the health and status of complex engineered systems". Furthermore, according to Sheppard et al. (2009) [8], PHM could be defined as "a maintenance and asset management approach utilizing signals, measurements, models, and algorithms to detect, assess, and track degraded health, and to predict failure progression". According to Chen et al. (2012) [9], PHM comes from the U.S. DoE (Department of Energy) and the U.S. DoD (Department of Defense). In the JSF (Joint Strike Fighter) program of U.S. DoD, the name PHM was adopted.

Regarding PHM term, there are some similar terms used interchangeably: e.g., predictive maintenance, prognostics, and CBM. Among them, the most well-known terminology is CBM. Over the last decade, CBM has evolved into the concept of PHM, due to its broader scope [7].

The purpose of PHM is to maximize the operational availability and safety of the engineering asset that eventually improves security, reliability, availability and mission success, and support a better ability to plan for maintenance events, which leads to reduce the operation and maintenance cost $[8,10,11]$. The PHM can be done by (1) gathering product status data; (2) monitoring the product 
condition based on gathered data; (3) making a diagnosis of a product status; (4) predicting the remaining useful life of the product; and (5) executing appropriate actions such as repair, replace, and disposal based on reasonable decision making.

In general, the PHM system should have the capability of anomaly detection, fault diagnostics, fault prognostics, and health management. To get the capability, various methods have been considered, which could be mainly grouped into physics-based, data-driven, and hybrid approaches.

According to Vichare et al. (2006) [5], Luna (2009) [12], Zhang et al. (2009) [6], Sun et al. (2012) [13], and Javed (2014) [7], the following are the benefits of PHM:

Table 1. Benefits of PHM

\begin{tabular}{l}
\hline Domain \\
\hline Quality \\
- Maintain effectiveness through timely repair actions, \\
- Improve system safety, \\
- Increase system operations reliability and mission availability, \\
- Increase availability, \\
- Avoid consequences of failure by advance warning of failures, \\
- Extend life/Reduce maintenance frequency, \\
- Optimize resource use, \\
- Decrease unnecessary maintenance actions, \\
- Improve system safety (predict to prevent negative outcomes), \\
- Improve qualification and assisting in the design and logistical support \\
- Improved and future systems, \\
- Reduce system LCC (Life-Cycle Cost) by decreasing inspection costs, \\
- downtime, and inventory, \\
- Reduce operational costs to optimized maintenance. \\
\hline Cost \\
- Minimize unscheduled maintenance, \\
- Extend maintenance cycles
\end{tabular}

\section{$3 \quad$ Previous works}

So far there have been several research works related to PHM or CBM or predictive maintenance. For example, Vichare and Pecht (2006) [5] presented the state-of-practice and the current state-of-research in the area of electronics PHM. In their work, examples for four approaches such as BIT (Built-In-Test), use of fuse and canary devices, monitoring and reasoning of failure precursors, and modeling accumulated damage based on measured life-cycle loads were provided. Luna (2009) [12] provided a framework for organizing themes of PHM benefits by identifying overall categories for understanding the different types of impacts and benefits a PHM system can have from a logistics support perspective. They mentioned the benefits of PHM from the logistics support perspective: 1) Reduce lead time, 2) Avoid consequences of failure, 3) Extend Life/Reduce Maintenance Frequency, and 4) Optimize resource use. Cheng et al. (2010) [14] introduced the state-of-the-art sensor systems for PHM and the emerging trends in technologies 
of sensor systems for PHM. They discussed the considerations for sensor system selection for PHM applications, including the parameters to be measured, the performance needs, the electrical and physical attributes, reliability, and cost of the sensor system. Das et al. (2011) [10] discussed the essential steps for an effective PHM system. In their work, they described time and frequency domain features that can be extracted from raw sensor data. They also described a case study of implementing a PHM system for a high speed face milling CNC cutter. Pecht (2012) [15] presented an assessment of the state of practice in PHM of information and electronics rich systems. He presented a fusion prognostics approach, which combines or fuses together the model-based and data-driven approaches, to enable markedly better prognosis of remaining useful life. In order to illustrate the implementation of the fusion approach to prognostics, he carried out a case study of a printed circuit card assembly. Recently, Lee et al. (2014) [16] carried out a comprehensive review of the PHM field. They introduced a systematic PHM design methodology, 5S methodology, for converting data to prognostics information. They also presented a systematic methodology for conducting PHM as applied to machinery maintenance.

\section{Prognostics applications}

Table 2. Case studies of prognostics

\begin{tabular}{llll}
\hline Equipment & Sensor type & no. of sensors & \multicolumn{1}{c}{ Approach } \\
\hline - Compressor & - Vibration & - Single & - Regression-based [17] \\
& & - Multiple & - Bayesian network-based [18] \\
\hline - Mooring line & - Tension & - Single & - RUL estimation based on k-means, \\
& & ARIMA, and Miner's rule [19] \\
\hline - Electric generator - Temperature - Single & - DTW-based approach \\
\hline
\end{tabular}

This section introduces three case studies for prognostics that we have carried out until now. Table 2 shows the summary of case studies. In Cho et al. (2016) [17], we have introduced two approaches predicting the next failure time or identifying failure status of the gas compressor which is one of essential mechanical equipment in both onshore and offshore plants, based on gathered vibration data: Regression-based approach (Cho et al. (2016) [17]), and Bayesian network-based approach (Jun and Kim (2017) [18]). Furthermore, we have dealt with estimating the RUL (Remaining Useful Life) of the mooring line of an offshore plant based on gathered ocean environment data and mooring line tension data. For the mooring line case, we have developed a hybrid approach integrating data-driven and physics-based approaches. In the proposed approach, not only data analyzing methods such as k-means clustering method and ARIMA, but also tension damage calculation method by the Miners rule has been applied to predict the RUL of the mooring line. For more details, please refer to Kim et al. (2016) [19]. In addition, we have developed an algorithm for estimating the 
RUL by analyzing the temperature sensor data of an electric generator using a DTW (Dynamic Time Warping) based approach.

Except the mooring line case, three cases are all data driven approaches. For the limited test data, the proposed approaches have been applied. As the result, we could see that the proposed approaches give some reliable results for prognosis. For example, in the case study of regression-based approach [17], we could see that the estimated next failure time is close to the real failure time. But, we could also find that the regression model based approach gave the poor performance when the remaining residual life time is relatively long [17]. In the Bayesian network based approach and DTW based approach, for the generated test examples, we could see that our methods give reasonable results. There must be some benefits in the plant industry from the plant operation and safety viewpoints since the suitable decision based on equipment prognosis result could be made, if this kind of PHM system is commercially applied in the real field. You may see the details of the test results in the relevant references [17-19].

\section{Limitations and discussion}

This study introduced the case studies of prognostics algorithm for plant equipment with the PHM concept. Although we have looked the possibility of PHM approach with several case studies, there are still some limitations in applying its concept as follows.

First, the core algorithms of the PHM are to diagnose the current state of equipment based on the collected data and to predict RUL in the future. The accuracy of these algorithms is mostly affected by the amount and quality of the gathered data. When implementing PHM, if there are much amount of failure data available, data-driven algorithms could be applied. However, in reality, it was difficult to get the actual failure history. In our study, the proposed approaches have been tested based on limited real failure data or the generated data based on it. Thus, to prove the robustness of the approach, further exhaustive test should be done based on lots of real data.

Second, another limitation is that most algorithms mentioned in this study dealt with the single variable or single location problem. As the result, there is a limit to precisely predict the state of the equipment and the time of failure with only a single variable sensor data at one location of the equipment. To resolve this problem, the Bayesian network based approach could be applied to more high-level equipment based on the integrated failure propagation model of the target plant equipment to identify the multiple causes and failures in an integrated way.

With these limitations, the following point could be discussed: We could think of applying the PHM into not only maintenance but also other domains, e.g., product design improvement. For example, we can build a system to improve the design by reflecting the diagnostics and prognostics results of the PHM as well as the maintenance of the equipment by creating a closed link between design improvement and the PHM. The RUL value estimated at a certain time 
can be compared to the theoretical RUL of a product, calculated according to the difference between the designed lifetime and the equipment operating time. A comparison of these values tells us how adequately the equipment is being used. As a result, if the RUL is longer than the theoretical RUL, we can then let the equipment go without any maintenance actions or modify the severity of the mission profile for use with more intensive applications. Otherwise, PHM operations should be done. This kind of information could provide us with the feedback for product design improvement.

In order to develop a more accurate prognostics algorithm in the future, the following points should be considered. First of all, the cause-symptom-failure relations should be well defined for each equipment. To this end, the well-designed modeling method to represent this relations should be developed. Second, a clear definition of failure and a criterion for determining the failure of equipment, for example, thresholds, should be defined. Third, failure history data, sensor signal data at the time of failure, and operation related data should be integrated and continuously recorded and managed. Finally, when the equipment is operated at the beginning of its life, there will be only a few failure data, which leads to make estimating the RUL of the equipment impossible with considered prognostics algorithms. Therefore, it is needed to estimate the RUL in a different way. For example, in the early life stage of equipment, it is reasonable to estimate the RUL based on the reliability data provided by manufacturers. Then, if the failure history data is accumulated systematically, we need to apply a learning-based algorithm that improves the accuracy of the RUL prediction.

\section{Conclusion}

In this study, we have briefly looked into PHM concept and prognostics case studies. The purpose of PHM is to maximize the operational availability and reliability of the engineering system. Using PHM technologies, the companies could have the ability to prevent the unexpected abnormality of the engineering system during its usage lifetime. In the era of the fourth industrial revolution, the PHM is becoming an important component of system design and operation of complex systems. PHM is not a single technique and requires very complex and various interdisciplinary research techniques, which leads to take much time and

efforts for PHM. Although this study did not introduce exhaustive and detailed PHM works, it could be a base for future PHM works.

\section{Acknowledgments}

This research was supported by Basic Science Research Program through the National Research Foundation of Korea(NRF) funded by the Ministry of Education (NRF-2017R1D1A1B03031633). 


\section{References}

1. Shin, J.H., Jun, H.B.: On condition based maintennace policy. Journal of Computational Design and Engineering 2, 119-127 (2015).

2. O'Donovan, P., Leahy, K., Bruton, K., O'Sullivan, D.T.J.: An industrial big data pipeline for data-driven analytics maintenance applications in large-scale smart manufacturing facilities. Journal of Big Data 2(25), 1-26 (2015).

3. Wang, S., Wan, J., Li, D., Zhang, C.: Implementing Smart Factgory of Industrie 4.0: An Outlook. International Journal of Distributed Sensor Networks, 1-10 (2016).

4. Xu, L.D., He, W., Li, S.: Internet of Things in Industries: A Survey. IEEE Transactions on industrial information 10(4), 2233-2243 (2014).

5. Vichare, N.M., Pecht, M.G.: Prognostics and Health Management of Electronics. IEEE Transactions on components and packaging technologies 29(1), 222-229 (2006).

6. Zhang, H., Kang, R., Pecht, M.: A hybrid prognostics and health management approach for condition-based maintenance. In: the 2009 IEEE conference proceedings, pp. 1165-1169 (2009).

7. Javed, K.: A robust and reliable data-driven prognostics approach based on Extreme Learning Machine and Fuzzy Clustering. Ph.D. Thesis. Université de Franche-Comté (2014).

8. Sheppard, J.W., Kaufman, M.A., Wilmering, T.J.: IEEE Standards for prognostics and health management. IEEE A\&E Systems Magazine, 34-41 (2009).

9. Chen, Z.S., Yang, Y.M., Hu, Z.: A technical framework and roadmap of embedded diagnostics and prognostics for complex mechanical systems in prognostics and health management systems. IEEE Transactions on Reliability 61(2), 314-322 (2012).

10. Das, S., Hall, R., Herzog, S., Harrison, G., Bodkin, M.: Essential steps in prognostic health management. In: IEEE conference on prognostics and health management proceedings, pp. 1-9, (2011).

11. Jinyu, Z., Xianxiang, H., Wei, C.: Research on Prognostic and Health Monitoring System for large complex equipment. In: IITA International Conference on Control, Automation and Systems Engineering proceedings, pp. 1-6, (2009).

12. Luna, J.J.: Metrics, models, and scenarios for evaluating PHM effects on logistics support. Annual Conference of the Prognostics and Health Management Society proceedings, (2009).

13. Sun, B., Zeng, S., Kang, R., Pecht, M.G.: Benefits and challenges of system prognostics. IEEE Transactions on Reliability 61(2), 323-335 (2012).

14. Cheng, S., Azarian, M.H., Pecht, M.G.: Sensor systems for prognostics and health management. Sensors 10, 5774-5797 (2010).

15. Pecht, M.: A prognostics and health management for information and electronicsRich systems. Engineering Asset Management and Infrastructure Sustainability, 19$30(2012)$.

16. Lee, J., Wu, F., Zhao, W., Ghaffari, M., Liao, L.: Prognostics and health management design for rotary machinery systems-reviews. Mechanical Systems and Signal Processing 42, 314-334 (2014).

17. Cho, S.J., Shin, J.H., Jun, H.B., Hwang, H.J., Ha, C.H., Hwang, J.S.: A study on estimating the next failure time of a compressor equipment in an offshore plant. Mathematical problems in Engineering, 1-14 (2016).

18. Jun, H.B., Kim, D.: A Bayesian network-based approach for fault analysis. Expert Systems with Applications 55(5), 1350-1367 (2017). 
19. Kim, Y.J., Seo, M., Jun, H.B., Ha, C.H., Shin, J.H.: A study on predictive algorithm for estimating the RUL of offshore plant equipment. In: TCIEAS proceedings, pp. $1-10,(2016)$. 